\title{
Structure of Nascent Microbial Cellulose V. Influence of Number of Sulfonate Group in Fluorescent Brightener on Crystal Structure of Microbial Cellulose
}

\author{
Akira $\mathrm{KAI}^{\dagger}$ and Sherif M. A. S. KeSHK \\ Department of Applied Chemistry, Tokyo Metropolitan University, \\ Minami-Ohsawa 1-1, Hachioji-shi, Tokyo 192-0397, Japan
}

(Received June 15, 1998)

\begin{abstract}
The influence of the number of sulfonate groups in a Fluorescent Brightener on the crystal structure of microbial cellulose was examined by X-ray diffraction and solid state ${ }^{13} \mathrm{C}$ NMR spectroscopy. All products obtained from Acetobacter xylinum-culture in the presence of a Fluorescent Brightener with 2 (FB), 4 (FWA2), or 6 sulfonate groups (FWA3) were crystalline complexes that contained a dye between cellulose sheets corresponding to the (110) plane of microbial cellulose. Cellulose regenerated from the FB complex forms a cellulose $\mathrm{I}_{\beta}$ rich structure, whereas cellulose regenerated from FWA2 and FWA 3 complexes form cellulose II. The influence of a Fluorescent Brightener on the crystal formation of regenerated microbial cellulose clearly differs according to the number of sulfonate groups in a dye. The nascent structure of microbial cellulose may be the structure that the conformation and arrangement of the cellulose chain changes when a dye adheres to it. KEY WORDS Acetobacter xylinum / Microbial Cellulose / Fluorescent Brightener / Sulfonate Group / Dye-Cellulose Complex / Cellulose $\mathrm{I}_{\beta} /$ Cellulose II /
\end{abstract}

When Acetobacter xylinum, one of bacteria that produces cellulose, is cultured in the presence of a direct dye, a crystalline complex containing a mono-molecular layer of a dye between the (110) planes of microbial cellulose is produced. ${ }^{1}$ When this complex is dye-extracted, cellulose, that does not exhibit the structure of ordinary microbial cellulose (cellulose $\mathrm{I}_{\alpha}$ rich structure) but cellulose $\mathrm{I}_{\beta}$ rich structure, cellulose $\mathrm{IV}_{\mathrm{I}}$ or II, is regenerated from the complex. ${ }^{1}$ The factor, that fixes the crystal type of cellulose regenerated from a dye cellulose complex, is likely the dye structure itself, and above all, the number of sulfonate groups per unit of molecular weight of the dye ${ }^{1}$ and its position. ${ }^{2}$ Cellulose $I_{V}$ is regenerated from the Direct Red 28cellulose complex, ${ }^{3}$ whereas cellulose II is regenerated from Direct Blue $1,14,15$, or 53 , with the same skeletal structure as Direct Red 28 and with 2 sulfonate groups more than Direct Red 28, and the cellulose complex. ${ }^{4,5}$ In the Direct Blue group, the dye does not only have a sulfonate group but also 1 hydroxy group and 1 methyl or methoxy group more than Direct Red 28. Therefore, the effect of a sulfonate group on the crystal structure of regenerated cellulose is not always definite.

In this study, we examined the effect of the number of sulfonate groups in a dye on the crystal structures of the complex and cellulose regenerated from it using a Fluorescent Brightener with 2 sulfonate groups (FB) and a Fluorescent Brightener with the same skeletal structure as FB except for 4 (FWA2) or 6 (FWA3) sulfonate groups. Furthermore, the structure of nascent microbial cellulose was considered from these results.

\section{EXPERIMENTAL}

\section{Preparation of Samples}

A. xylinum (IFO 13693) was cultured in a $15 \mathrm{~cm}$ diameter petridish which contains $110 \mathrm{ml}$ Hestrin-

\footnotetext{
† To whom correspondence should be addressed.
}

Schramm (HS) medium ${ }^{6}(\mathrm{pH} 6.8)$ at $28^{\circ} \mathrm{C}$ for about 3 days in an incubator. $60 \mathrm{ml}$ of cellulose-free cell suspension, prepared from matured petridish in $120 \mathrm{ml}$ phosphate buffer solution, were added to $140 \mathrm{ml}$ of $\mathrm{HS}$ medium ( $\mathrm{pH} 7.0$ ) containing a direct dye of $0.1 \mathrm{wt} \%$ and the mixture was incubated at $28^{\circ} \mathrm{C}$ for $24 \mathrm{~h}$ under a static condition. The product was collected by centrifuging $(6000 \mathrm{rpm}, 5.15 \mathrm{~g}, 20 \mathrm{~min})$. The product obtained from the medium containing FB was washed well with $0.1 \mathrm{wt} \%$ $\mathrm{NaOH}$ aqueous solution to remove the dye not related to dyeing and with distilled water until alkali-free. On the other hand, the product obtained in the presence of FWA 2 or FWA 3 was rinsed only with distilled water for 60 or $10 \mathrm{~min}$, respectively. These products were preserved at about $3^{\circ} \mathrm{C}$ in the wet state. The direct dyes used in this experiments are FB (4,4'-bis[4-anilino-6-bis(2-hydroxylethyl)amino-1,3,5-triazin-2-ylamino]-2,2'-stilbene disulfonate, Nippon Kayaku), FWA2 (tetrasodium 4,4'-bis $\{4$-bis(2-hydroxyethyl)amino-6-(4-sulfonatophenylamino)-1,3,5-triazin-2-ylamino $\}$ stilbene-2,2' -disulfonate, Nippon Kayaku), and FWA3 (hexasodium 4,4'-bis[4-bis(2-hydroxyethyl)amino-6-(3,6-disulfonatephenylamino)-1,3,5-triazin-2-ylamino)] stilbene-2,2' -disulfonate, Nippon Kayaku). The chemical structure of these dyes are shown in Figure 1.

The dye extraction of the above samples was performed by boiling in $70 \mathrm{vol} \%$ aqueous ethanol solutions for $18 \mathrm{~h}$ and the fresh aqueous ethanol solution was exchanged every $3 \mathrm{~h}$. This dye extracted sample was again boiled in $1.0 \mathrm{wt} \%$ aqueous sodium hydroxide solution for $10 \mathrm{~h}$ under $\mathrm{N}_{2}$ atmosphere to remove protein. This regenerated cellulose sample was neutralized with $1.0 \mathrm{vol} \%$ aqueous acetic acid and subsequently, rinsed with distilled water. The sample was preserved as above.

Control cellulose I sample was prepared by washing microbial cellulose with distilled water to remove the medium components and then, was boiled in $1.0 \mathrm{wt} \%$ aqueous sodium hydroxide solution for $10 \mathrm{~h}$ under $\mathrm{N}_{2}$ atmosphere. The final sample was neutralized and rinsed 


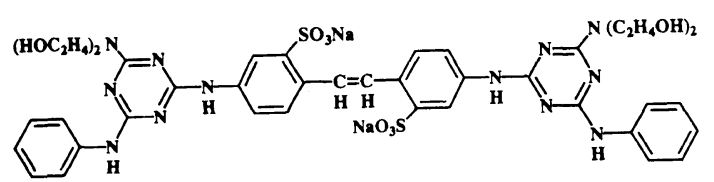

FB
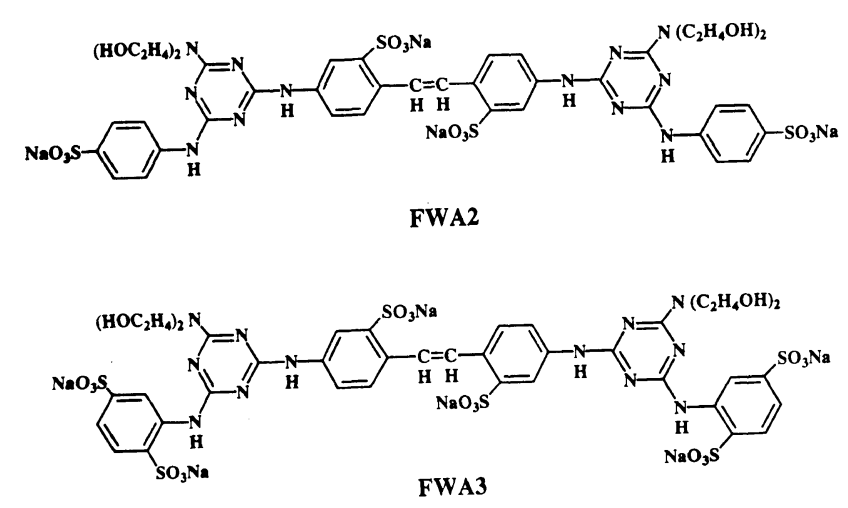

Figure 1. The chemical structure of direct dyes.

as above. The cellulose II sample was prepared by mercerization of microbial cellulose at $20^{\circ} \mathrm{C}$ for $1 \mathrm{~h}$.

\section{Measurements}

In order to understand the structures of the product from Acetobacter-culture in the presence of FB, FWA2, or FWA3 and of the cellulose regenerated from it, the following meaurements were performed. The X-ray diffractogram of an uniplanar oriented membrane of a product was measured with the reflection and transmission methods. The membrane was prepared from the preserved product and was dried on a Teflon plate at room temperature. On the other hand, the diffractogram of an isotropic product and its regenerated cellulose was performed by the transmission method. In this method, the sample was compressed as a pellet into the pore of a sample holder of $2 \mathrm{~mm}$ diameter and $1 \mathrm{~mm}$ thickness. The X-ray diffractogram was measured by $\mathrm{MXP}^{18}$ diffractometer (MAC Science) with Ni-filtered $\mathrm{Cu}-K_{\alpha}$ radiation. The measurement conditions were as follows: divergence slit, $1.0 \mathrm{~mm}$; receiving slit, $0.15 \mathrm{~mm}$; scanning speed, $4.0^{\circ} \mathrm{min}^{-1}$; X-ray radiation, $40 \mathrm{kV}, 200 \mathrm{~mA}$.

Solid state ${ }^{13} \mathrm{C}$ NMR experiments were performed at room temperature on a JEOL JNM EX 270 spectrometer operating at $6.35 \mathrm{~T}$. The MAS rate was $5.0-5.5 \mathrm{kHz}$, and the chemical shifts relative to tetramethylsilane were determined by using the $\mathrm{CH}_{3}$ peak at $17.3 \mathrm{ppm}$ of solid hexamethyl benzene as an external standard. The non-dried samples were packed in a MAS rotor with an O-ring seal to avoid the loss of water during NMR measurements. The spectrum of the crystalline component was measured selectively by taking NMR signal after a relaxation of the noncrystalline component. Therefore, in order to know the crystalline component of control and regenerated celluloses, the signal obtained after $\tau=50 \mathrm{~s}$ was measured by using CPT1 pulse sequence $^{7}$ with CP except for microbial cellulose and cotton. The spectra of microbial cellulose and cotton were quoted from previous paper ${ }^{8}$.

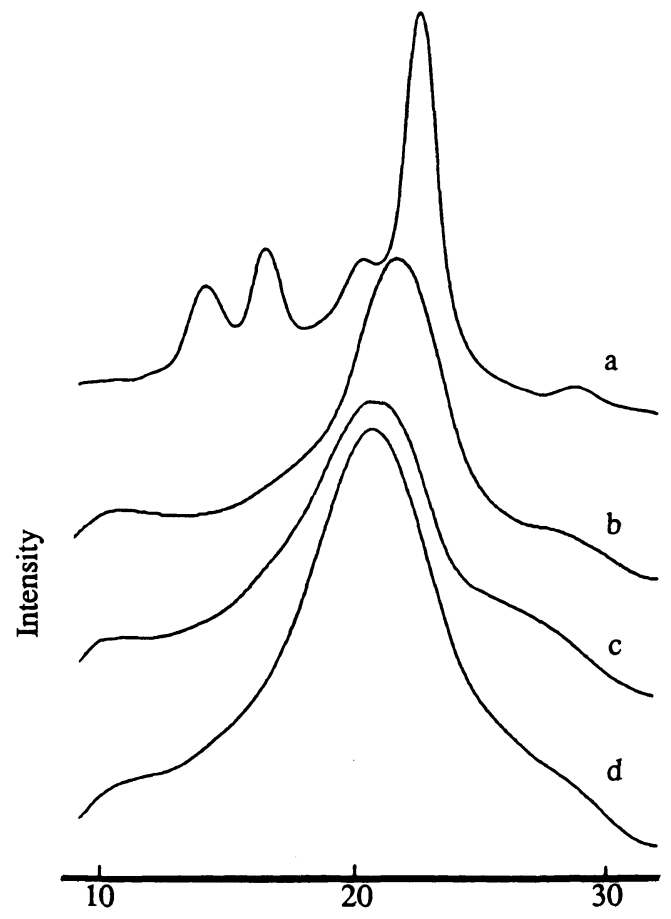

$2 \theta\left({ }^{\circ}\right)$

Figure 2. X-Ray diffractograms of isotropic samples of microbial cellulose and the product samples from Acetobacter-culture in the presence of a dye of $0.1 \mathrm{wt} \%$. a, microbial cellulose; b, FB product; c, FWA2 product; d, FWA3 product.

Table I. X-Ray diffraction angles of MC and products obtained from Acetobacter-culture in the presence of FB, FWA2, or FAW3

\begin{tabular}{llllll}
\hline Sample & \multicolumn{5}{c}{$2 \theta / \mathrm{deg}$} \\
MC & & 14.5 & 16.8 & 20.1 & 22.7 \\
FB & 10.8 & & & 20.7 & 21.7 \\
FWA2 & 11.0 & & & 20.8 & \\
FAW3 & 11.0 & & & & \\
\hline
\end{tabular}

MC, microbial cellulose.

\section{RESULTS AND DISCUSSION}

Figure 2 shows X-ray diffractograms of isotropic samples of microbial cellulose and the products (FB product, FWA2 product, and FWA3 product) obtained from A. xylinum culture in the presence of FB, FWA2, or FWA3. Table I shows $2 \theta$ s of diffraction peaks in the diffractograms shown in Figure 2. Figure 3 shows $X$ ray diffractograms of FB, FWA2, and FWA3 product membranes measured by the transmission and reflection methods. Table II shows $2 \theta$ s of diffraction peaks in Figure 3.

As shown in Figure 2, all products obtained from $A$. xylinum culture in the presence of FB, FWA2, or FWA3 showed the characteristic diffractogram with 2 diffraction peaks. In the diffractograms of the FB product, 2 diffraction peaks occurred at 10.8 and $21.7^{\circ}$, whereas in the diffractogram of FWA2 and FWA 3 products, diffraction peaks were observed near 11.0 and $20.7^{\circ}$, respectively. These product diffractograms differed from that with 4 peaks for microbial cellulose. In addition, 


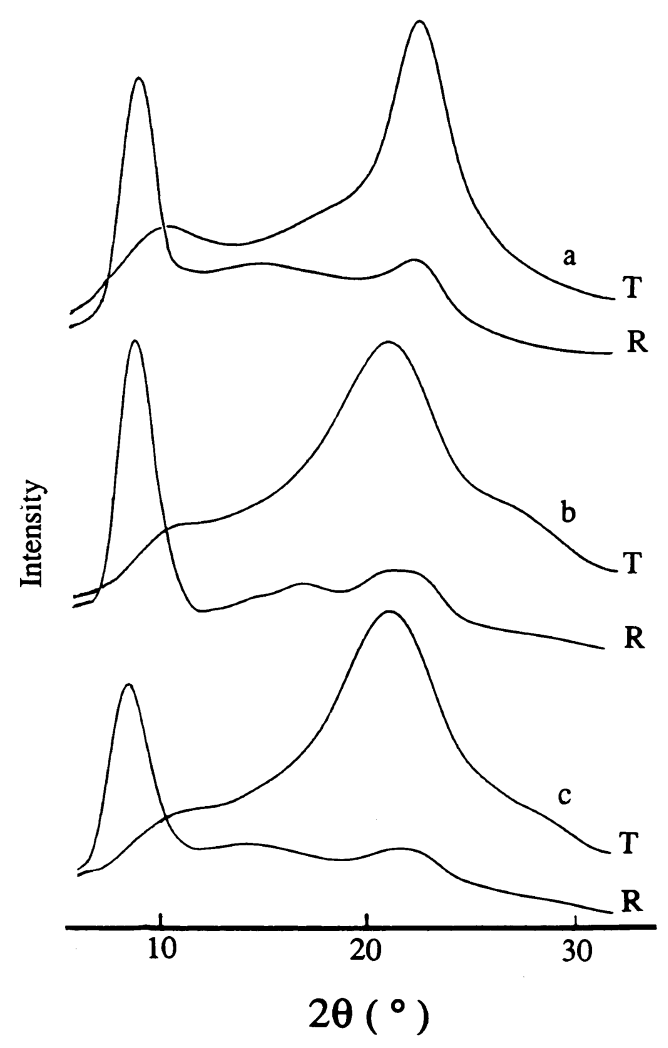

Figure 3. X-Ray diffractograms of the product membranes measured by the transmission (T) and reflection (R) methods. a, FB product; $b$, FWA2 product; c, FWA3 product.

Table II. X-Ray diffraction angles of FB, FWA1, and FWA2 products measured by transmision $(\mathrm{T})$ and reflection $(\mathrm{R})$

\begin{tabular}{llrr}
\hline \multicolumn{1}{c}{ Sample } & \multicolumn{3}{c}{$2 \theta / \operatorname{deg}(d$-spacing in $\AA)$} \\
\hline FB product & $\mathrm{T}$ & $10.3(8.61)$ & $22.3(3.98)$ \\
& $\mathrm{R}$ & $8.9(9.88)$ & $22.1(4.02)$ \\
FWA2 product & $\mathrm{T}$ & $10.4(8.52)$ & $20.6(4.03)$ \\
& $\mathrm{R}$ & $8.7(10.1)$ & $21.2(4.18)$ \\
FWA3 product & $\mathrm{T}$ & $10.8(8.01)$ & $20.8(4.26)$ \\
& $\mathrm{R}$ & $8.4(10.5)$ & $21.5(4.14)$ \\
\hline
\end{tabular}

these diffractograms were almost the same as those for products obtained from the culture in the presence of other direct dyes (Direct Red 28, 75, 79, 80, Direct Blue $1,14,15,53,71$, or Direct Brown 106) $)^{1,3-5}$ considering diffractograms with 2 diffraction peaks. The diffraction peak at the high angle side of the product in the presence of FB, Direct Red $28,{ }^{3}$ Direct Blue $71,{ }^{1}$ or Direct Brown $106^{1}$ occurred at $22-23^{\circ}$, whereas that of the product in the presence of FWA2, FWA3, or others occurred at $20-21^{\circ}$.

As shown in Figure 3 and Table II, in the diffractograms of the uniplanar oriented membrane of the FB product measured by the transmission method with the X-ray beam which was perpendicularly transmitted to the surface of the membrane, weak and strong peaks occurred at 10.3 and $22.3^{\circ}$, respectively. However, in the diffractogram obtained by the reflection method with the X-ray beam which was reflected to the surface of the membrane, strong and weak peaks occurred at 8.9 and $22.1^{\circ}$, respectively. In the diffractogram of the FWA 2 and FWA 3 products mem-

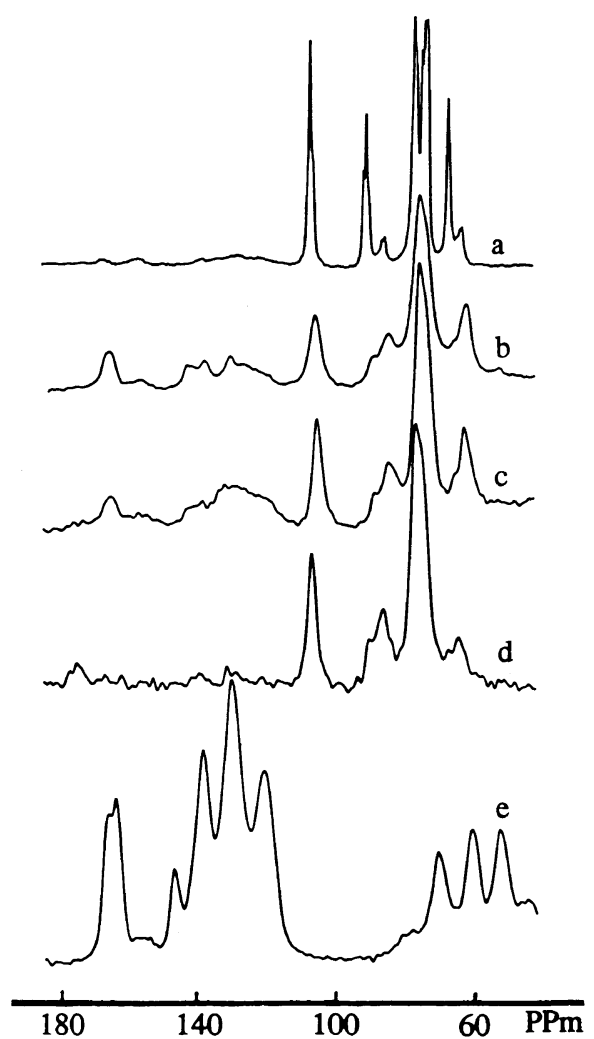

Figure 4. Solid state ${ }^{13} \mathrm{C}$ NMR spectra of wet microbial cellulose, wet products, and dry direct dye. a, microbial cellulose; b, FB product; c, FWA2 product; d, FWA3 product; e, FWA3 powder.

brane measured by the transmission method, weak and strong peaks occurred near 10.6 and $20.7^{\circ}$, respectively. In the case of the reflection method, strong and weak peaks occurred near 8.5 and $21.3^{\circ}$, respectively. The diffraction planes at the low angle side of these product membranes were parallel to its surface, whereas the diffraction planes at high angle side were perpendicular to the surface.

The diffraction plane at the low angle side must correspond to the plane that expands by the inclusion of a dye between cellulose sheets corresponding to the (110) plane at $14.5^{\circ}$ for microbial cellulose as previouly described. ${ }^{9}$ Namely, this plane corresponded to the (100) plane of the complex composed of a dye and cellulose. ${ }^{3,4}$ In contrast, the diffraction plane observed near $21^{\circ}$ must correspond to the $(010)$ of the complex, ${ }^{3,4}$ The structures of the FB, FWA2, and FWA3 products were similar because a dye was included between cellulose sheets.

Figure 4 shows solid state ${ }^{13} \mathrm{C}$ NMR spectra of $\mathrm{FB}$, FWA2, and FWA3 complexes, FWA3 powder and microbial cellulose. The chemical shifts of resonance lines of each sample are listed in Table III.

As shown in Figure 4, no resonance lines of the cellulose component in the FWA3 complex overlapped the line of the dye powder except for the C6 resonance line. However, the dye content of the FWA 3 complex was very low. It is therefore possible to discuss the structure of the cellulose component in the complexes using the $\mathrm{CP} / \mathrm{MAS}{ }^{13} \mathrm{C}$ NMR spectra. No resonance lines of the cellulose component in the FB and FWA2 complexes overlapped the lines of respective dye powders previously reported. ${ }^{2,8}$ 
Table III. ${ }^{13} \mathrm{C}$ chemical shifts ( $\mathrm{ppm}$ ) of microbial cellulose and products obtained from Acetobacter-culture in the presence of FB, FWA1, or FWA 2

\begin{tabular}{lcccc}
\hline \multicolumn{1}{c}{ Sample } & C1 & C4 & C2,3,5 & C6 \\
\hline MC & 106.3 & $90.2,84.7$ & $75.9,73.8,72.3$ & $66.5,62.5$ \\
FB product & 105.3 & -84.5 & 74.7 & -62.5 \\
FWA2 product & 105.0 & -84.1 & 74.3 & -63.0 \\
FWA3 product & 106.0 & -84.4 & 74.7 & -63.0 \\
\hline
\end{tabular}

MC, microbial cellulose.

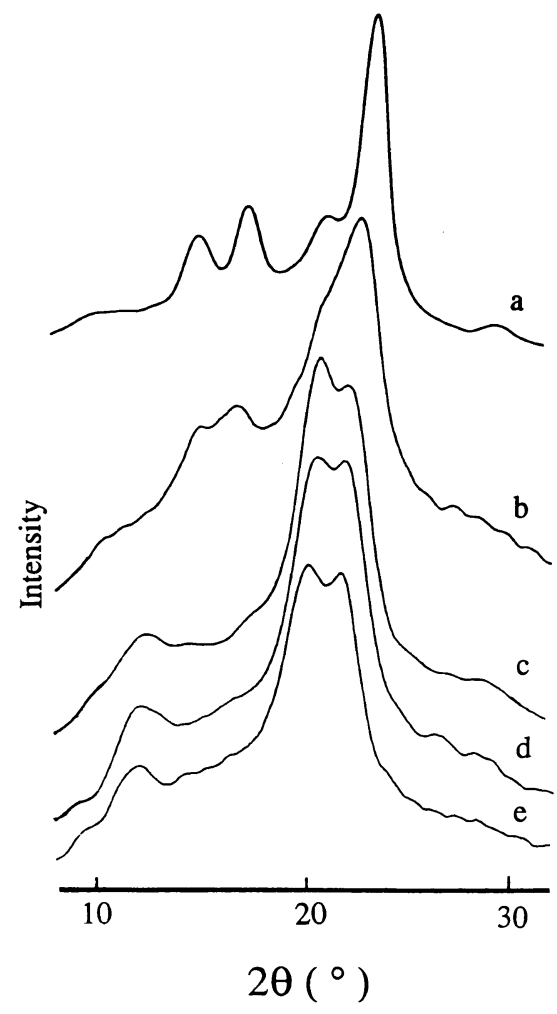

Figure 5. X-Ray diffractograms of the celluloses regenerated from the product samples. a, microbial cellulose; b, FB cellulose; c, cellulose II; d, FWA2 cellulose; e, FWA3 cellulose.

As shown in Figure 4 and Table III, the resonance lines of each carbon of the cellulose component in all complexes were broader than those of microbial cellulose. Moreover, the C4 and C6 resonance lines for the complexes were near 85.0 and $62.5 \mathrm{ppm}$, respectively. Furthermore, the $\mathrm{C} 1$ resonance line of each complex was shifted upfield by $0.3-1.3 \mathrm{ppm}$ compared to that of microbial cellulose. Although X-ray mesurements indicated that the complex was in a crystalline state, solid state ${ }^{13} \mathrm{C}$ NMR spectroscopy indicated that the cellulose component in the complex was in a noncrystalline state. This discrepancy suggests that the mobility of the carbon of the cellulose component in the complex is almost the same level as for the carbon in the noncrystalline region of microbial cellulose because hydrogen bonding between cellulose sheets is hindered by the inclusion of a dye between sheets in the complex. The spectrum of the cellulose component in each FB, FWA2, and FWA3 complex was similar, possibly because a dye was included between cellulose sheets.

Figure 5 shows X-ray diffractograms of isotropic samples of cellulose regenerated from complexes (FB
Table IV. X-Ray diffraction angles of microbial cellulose, cellulose II, and celluloses regenerated from products

\begin{tabular}{lccc}
\hline \multirow{2}{*}{ Sample } & \multicolumn{3}{c}{$2 \theta / \mathrm{deg}$} \\
\cline { 2 - 4 } & $(1 \overline{1} 0)$ & $(110)$ & $(020)$ \\
\hline MC & 14.5 & 16.8 & 22.7 \\
FB cellulose & 14.4 & 16.6 & 22.3 \\
Cellulose II & 12.0 & 19.8 & 21.1 \\
FWA2 cellulose & 11.7 & 20.1 & 21.6 \\
FWA3 cellulose & 12.0 & 20.2 & 21.7 \\
\hline
\end{tabular}

MC, microbial cellulose.

cellulose, FWA2 cellulose, and FWA3 cellulose), microbial cellulose and cellulose II, and $2 \theta$ s of the (11 0$)$, (110), and (020) planes of each sample are listed in Table IV.

As shown in Figure 5 and Table IV, cellulose regenerated from the FB complex formed cellulose I. In contrast, cellulose regenerated from FWA2 and FWA3 complexes formed cellulose II.

Judging from $\mathrm{X}$-ray diffraction and solid state ${ }^{13} \mathrm{C}$ NMR spectroscopy of the complex, the structures of FB, FWA2, and FWA3 complexes were similar. However, the conformation and the arrangement of cellulose chains in a cellulose sheet may be different between the FB complex and FWA 2 and FWA 3 complexes. In fact, in X-ray diffractograms of these complexes, $2 \theta$ of the diffraction peak at the high angle side differed by about $2^{\circ}$ between the FB complex and FWA2 and FWA3 complexes. Cellulose regenerated from the complex in which the diffraction peak at the high angle side occurred at $22-23^{\circ}$ formed cellulose $\mathrm{I}$ or $\mathrm{IV}_{\mathrm{I}}^{3}$, while cellulose regenerated from the complex groups with the diffraction peaks at $20-21^{\circ}$, formed cellulose II.

Solid state ${ }^{13} \mathrm{C}$ NMR spectra of the crystalline component of each regenerated cellulose, microbial cellulose, cotton and cellulose II are shown in Figure 6.

As shown in Figure 6, the spectrum of FB cellulose did not resemble that of microbial cellulose with a cellulose $\mathrm{I}_{\alpha}$ rich structure but resembled that of cotton with a cellulose $\mathrm{I}_{\beta}$ rich structure. In contrast, the spectra of FWA2 and FWA3 celluloses distinctly showed the spectrum of cellulose II. The effect of FB with 2 sulfonate groups on the crystal structure of regenerated microbial cellulose clearly differed from that of FWA2 with 4 sulfonate groups and FWA3 with 6 sulfonate groups.

If the dye molecular weight was greater than 300 per sulfonate group (MW/N: MW, dye molecular weight; $\mathrm{N}$ : number of sulfonate group), cellulose $\mathrm{I}$ or $\mathrm{IV}_{\mathrm{I}}{ }^{3}$ was regenerated from the dye-cellulose complex, whereas, if it was less than 300 , cellulose II was regenerated. ${ }^{1} \mathrm{MW} / \mathrm{N}$ values were 458, 262, and 234 for FB, FWA2, and FWA3, respectively.

With an MW/N greater than 300 , because the solubility of a dye toward water becomes lower and the affinity of a dye toward cellulose becomes strong, the dye may protect the structure of the cellulose sheet, especially, in the conformation of cellulose chains. However, cellulose regenerated from the FB complex did not form the cellulose $\mathrm{I}_{\alpha}$ rich structure of ordinary microbial cellulose 


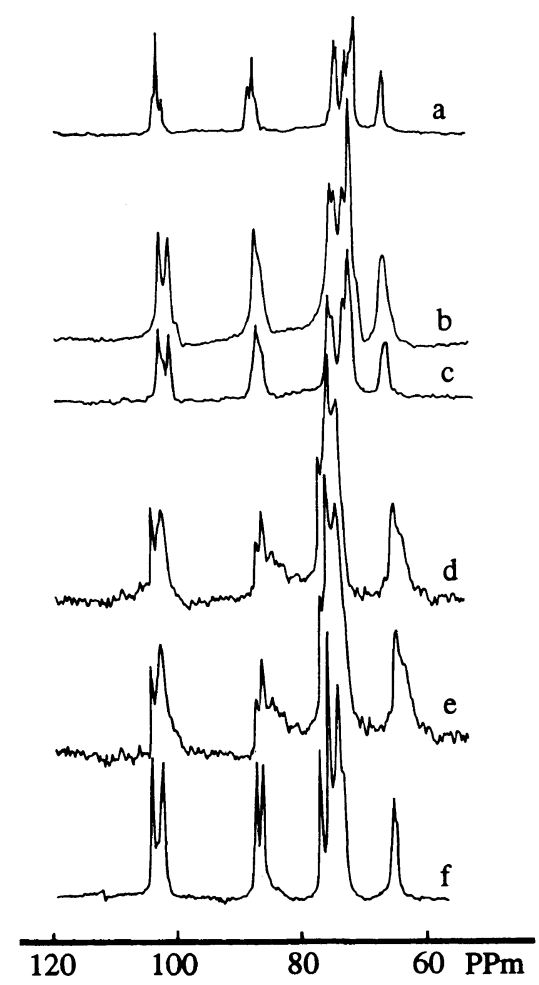

Figure 6. Solid state ${ }^{13} \mathrm{C}$ NMR spectra of crystalline components in the wet cellulose regenerated from products, wet microbial cellulose, wet cotton, and cellulose II. a, microbial cellulose ${ }^{6} ; \mathrm{b}, \mathrm{FB}$ cellulose; $\mathrm{c}$, cotton $^{6}$; d, FWA2 cellulose; e, FWA3 cellulose; f, cellulose II.

but formed the cellulose $\mathrm{I}_{\beta}$ rich structure. Horii et al. suggested that a cellulose $\mathrm{I}_{\alpha}$ rich structure forms when cellulose is crystallized under shear stress along the molecular axial direction. ${ }^{10}$ As cellulose produced in the presence of a dye relaxes through the absorption and desorption processes of a dye toward cellulose, cellulose $\mathrm{I}_{\beta}$, which is thermodynamically more stable than cellulose $\mathrm{I}_{\alpha}$, may be formed without an overall change in conformation. In contrast, FWA2 and FWA3, which are highly water-soluble and show low affinity toward cellulose, may disrupt the conformation and arrangement of the cellulose chain when it adheres to nascent noncrystalline cellulose.

\section{CONCLUSIONS}

The products obtained from $A$. xylinum culture in the presence of FB with 2 sulfonate groups, FWA2 with 4 sulfonate groups or FWA3 with 6 sulfonate groups were crystalline complexes containing a mono-molecular layer of a dye between the (110) planes of microbial cellulose. Cellulose $\mathrm{I}_{\beta}$ rich cellulose was regenerated from the FB complex, whereas cellulose II was regenerated from the FWA 2 or FWA 3 and cellulose complexes. If the MW/N of a dye was greater than 300 , cellulose regenerated from the complex formed cellulose I, and if it was less than 300 , regenerated cellulose formed cellulose II.

Acknowledgments. The authors are grateful to Nippon Kayaku Co., Ltd. for gifts of Fluorescent Brightener (FB, FWA2, and FWA3).

\section{REFERENCES}

1. H. Kido and A. Kai, Polym. J., 30, 508 (1998).

2. A. Kai and S. M. A. S. Keshk, Polym. J., in press.

3. A. Kai and Md. I. H. Mondal, Int. J. Biol. Macromol., 20, 221 (1997).

4. Md. I. H. Mondal and A. Kai, Polym. J., 30, 78 (1998).

5. Md. I. H. Mondal and A. Kai, Polymer J., 30, 84 (1998).

6. S. Hestrin and M. Schramm, Biochem. J., 58, 345 (1954).

7. D. A. Torchia, J. Magn. Reson., 30, 613 (1978).

8. A. Kai, P. Xu, F. Horii, and S. Hu., Polymer, 35, 75 (1994).

9. A. Kai, Makromol. Chem. Rapid Commun., 5, 307 (1984).

10. H. Yamamoto, F. Horii, and A. Hirai, Cellulose, 3, 229 (1996). 